\title{
Evaluation of Symptom Heterogeneity in Neuropathic Pain Using Assessments of Sensory Functions
}

\author{
Kathrin Arning and Ralf Baron \\ Division of Neurological Pain Research and Therapy, Department of Neurology, Christian-Albrechts-Universität Kiel, Germany
}

\begin{abstract}
Summary: Classification of neuropathic pain has been based on disease entities, anatomical localization, or histological observations. Over the past decade, there has been an explosion in our understanding of the basic mechanisms of neuropathic pain. The exciting advances in basic science are paralleled by the recognition from clinical investigations that neuropathic pain is not a monolithic entity, but instead presents as a composite of pain and other sensory symptoms. Attempts are under way to supplement the traditional classification with a classification that links pain and sensory symptoms with neurobiological mechanisms. This mechanism- or symptom-based classification takes both negative and positive sensory symptoms into account. By using a battery of several standardized quantitative sensory tests, the characteristic profile of sensory symptoms
\end{abstract}

can be elucidated in each patient. Moreover, in questionnaires the verbal descriptors can depict the quality and intensity of the individual pain. The approach of classifying and subgrouping patients with neuropathic pain on the basis of symptoms or signs opens up new possibilities for stratifying patients in clinical trials. First, in clinical proof-of-concept trials the study population can be enriched prospectively on the basis of entry criteria defined a priori. This enrichment with patients who potentially require a specific treatment should increase the likelihood for positive trial outcomes. Second, in clinical practice it becomes possible to establish an individualized therapy-that is, to identify the particular patients who require a specific treatment option. Key Words: Neuropathic pain, questionnaires, mechanism-based classification, symptom profiles.

\section{INTRODUCTION}

Chronic pain is the reason for $40 \%$ of all visits to a general practitioner, causes severe suffering for many patients and presents a challenge to the doctor in terms of adequate diagnostic work-up and management. There are two broad categories of chronic pain conditions: nociceptive pain, which occurs after tissue disease or damage but in the presence of a functionally intact sensory nervous system (e.g., osteoarthritis), and neuropathic pain, which arises when the afferent nervous system itself is diseased or damaged ${ }^{1}$ (e.g., postherpetic neuralgia). In addition to nociceptive or neuropathic types, other chronic pain disorders should be distinguished, such as somatoform pain disorders, pain associated with psychoses, or pain sine materia (diagnosed by exclusion), which are devoid of evidence for any nociceptive and neuropathic processes.

\footnotetext{
Address correspondence and reprint requests to: Prof. Dr. med. Ralf Baron, Division of Neurological Pain Research and Therapy, Department of Neurology, Christian-Albrechts-Universität Kiel, Arnold-Heller-Str. 3, Haus 41, 24105 Kiel, Germany. E-mail: r.baron@ neurologie.uni-kiel.de.
}

\section{ETIOLOGY-BASED CLASSIFICATION}

It is common clinical practice to classify neuropathic pain according to the underlying etiology of the disorder and the anatomical location of the specific lesion. ${ }^{2}$ Accordingly, the majority of patients with painful lesions in the nervous system fall into three broad classes (Table 1). ${ }^{3}$

1) Neuropathic pain after peripheral nerve lesions. Painful peripheral neuropathies may be of traumatic, ischemic, inflammatory, toxic, metabolic, or hereditary etiology. The anatomical distribution pattern of the affected nerves provides valuable differential diagnostic clues as to possible underlying causes. Painful peripheral neuropathies are therefore grouped into symmetrical generalized polyneuropathies (diseases affecting many nerves simultaneously) and asymmetrical neuropathies (with a focal or multifocal distribution, or processes affecting the brachial or lumbosacral plexuses).

2) Neuropathic pain after lesions in the central nervous system. Central neuropathic pain syndromes 
Table 1. Disease- and Anatomy-Based Classification of Neuropathic Pain Syndromes

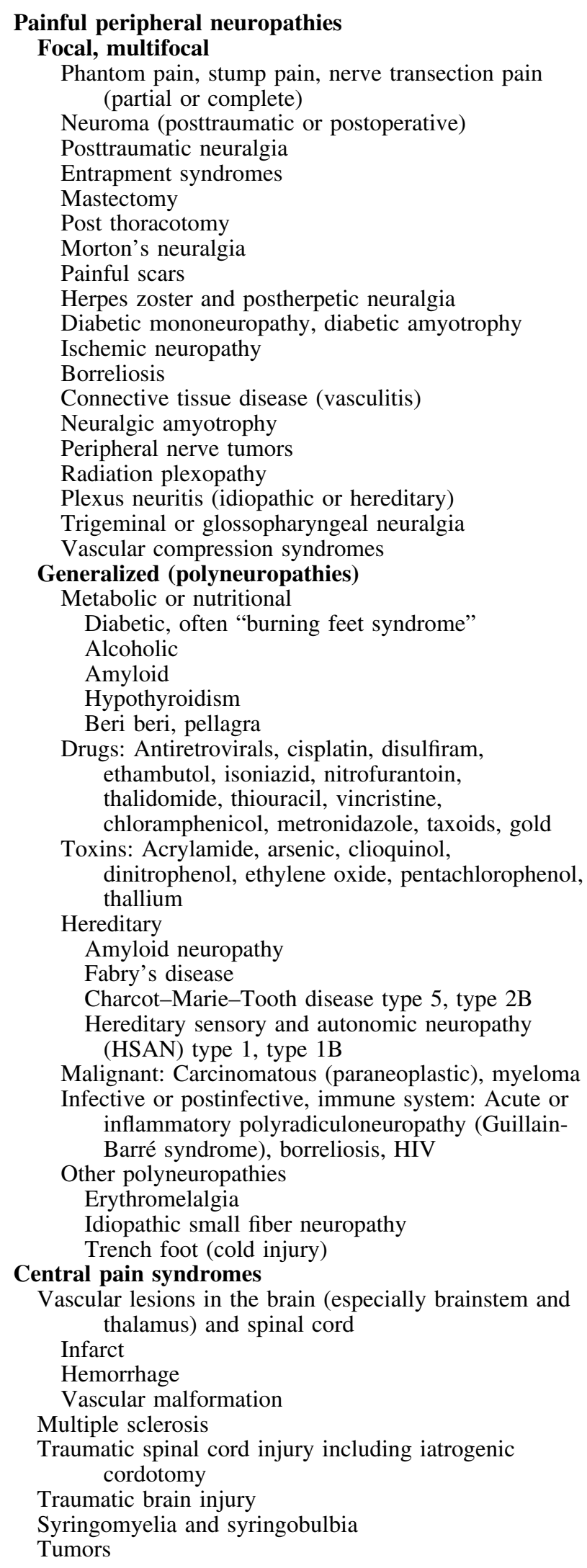

Table 1. Continued

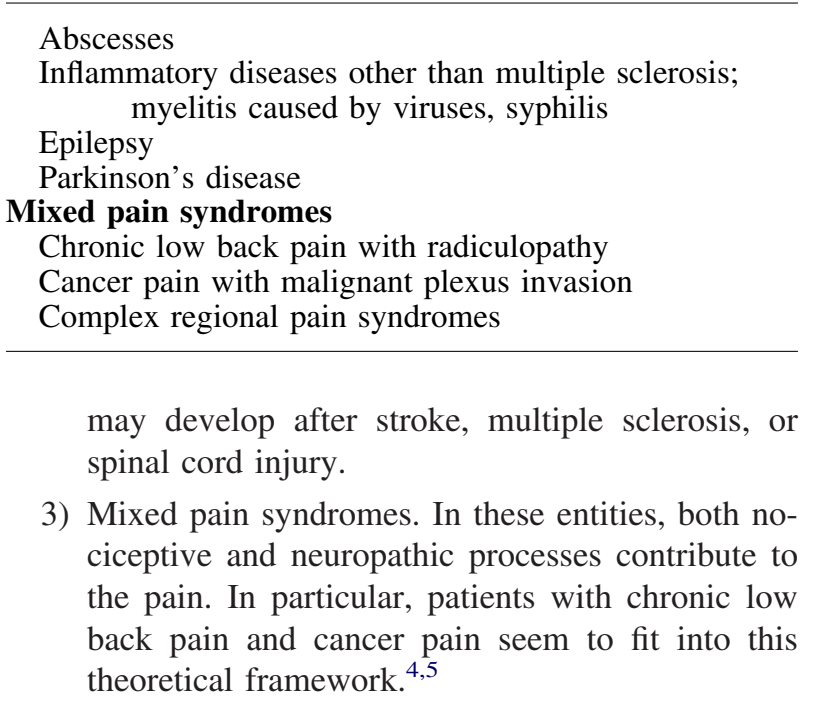

\section{RATIONALE FOR A NEW CLASSIFICATION}

For centuries, clinicians have been taught to examine and classify patients on the basis of the topography of the lesion and the underlying pathology. In most clinical specialties, such an approach has been a key element in understanding the pathophysiology of diseases and has led to progress in terms of finding disease-modifying or even disease-curing therapies. There are multiple examples, including bacterial meningitis, neuroborreliosis, osteoarthritis, cancer, rheumatoid arthritis, ischemic heart disease, and stroke. In most of these disorders, pain can be a major complaint, a symptom that rapidly disappears after the relevant therapy has been administered.

But what happens if the symptom itself becomes a disease? When pain persists and becomes a chronic problem, and when the underlying disease such as diabetes, cancer or vasculitis is known and cannot be cured? Clinical experience and decades of rather discouraging systematic research related to therapy in chronic pain have shown that a strategy directed at examining, classifying, and treating pain on the basis of anatomy or underlying disease is of little or no help to the patients and their pain. ${ }^{6}$ This has become desperately clear in some recent randomized clinical trials in painful diabetic neuropathy. In these trials, the study medications failed to demonstrate efficacy even though the compounds had shown encouraging results in preclinical and early clinical studies. ${ }^{7}$ Do these negative results really indicate lack of efficacy? Or is it possible that specific characteristics of the study population, the selected patient group, or the defined primary outcome have obscured a positive response? ${ }^{8}$

These observations have raised the question of whether an entirely different strategy, in which pain is analyzed on the basis of underlying mechanisms, could 
be an alternative approach to examining and classifying patients to obtain a better outcome. Increasing understanding of mechanisms underlying chronic pain, together with the discovery of new molecular targets for modifying pain, has strengthened the demand for other ways to treat pain. Woolf and other authors ${ }^{3,9}$ have emphasized the rationale for a treatment approach directed at one or more mechanisms rather than at diseases because new treatments are being developed on the basis of the biological mechanisms that underlie pain. One area that needs such a new approach is neuropathic pain.

Mechanisms of pain generation cannot be readily examined in the human patient. Notwithstanding, the expression of sensory abnormalities, the somatosensory phenotype, reveals some clues of pathophysiological dysfunctions of afferent processing. It is likely that certain pathophysiological mechanisms are responsible for characteristic signs of hypo- and hypersensitivity to mechanical and thermal stimuli, which can occur in many combinations. Some patients experience spontaneous pain, dysesthesias, and electric shocks, whereas in others the affected body area is hypersensitive to temperature or touch. $^{3}$

Thus, it might be the most appropriate approach to classify and subgroup patients with neuropathic pain according to their somatosensory phenotype. It is very likely that these distinct subgroups of patients will also respond differently to treatment. Consequently, patients in clinical trials should be stratified on the basis of this new classification scheme; that is, they should be grouped based on the phenotypic profile, rather than on the underlying etiology. This approach has the potential to minimize pathophysiological heterogeneity within the groups under study and to increase the power to detect a positive treatment result.

\section{CHARACTERISTIC SENSORY SIGNS AND SYMPTOMS IN NEUROPATHIC PAIN}

Pain associated with nerve injury has a number of clinical characteristics ${ }^{3}$ (Table 2). If a mixed peripheral nerve with a cutaneous branch or a central somatosensory pathway is involved, there is almost always an area of abnormal sensation, and the patient's maximum pain is coextensive with or within an area of sensory deficit. This is a key diagnostic feature for neuropathic pain. The sensory deficit often includes noxious and thermal stimuli, indicating damage to small-diameter afferent fibers or to the spinothalamic tract.

Beside these negative somatosensory signs (deficit in function), positive signs also are characteristic for neuropathic conditions. Paresthesias (ant crawling, tingling) are bothersome but not painful. Painful positive signs are spontaneous (i.e., not stimulus induced) ongoing pain and spontaneous shooting, electric-shock-like sensations.
Many patients with neuropathic pain also have evoked types of pain (stimulus-induced pain, hypersensitivity), which are characterized by several sensory abnormalities. They may be adjacent to or intermingled with skin areas of sensory deficit. Patients most often report mechanical hypersensitivity, followed by hypersensitivity to heat and cold.

Two types of hypersensitivity can be distinguished. First, allodynia is defined as pain in response to a nonnociceptive stimulus. In case of mechanical allodynia, even gentle mechanical stimuli may evoke severe pain. Second, hyperalgesia is defined as an increased pain sensitivity to a nociceptive stimulus. Another evoked feature is summation, which is the progressive worsening of pain evoked by slow repetitive stimulation with mildly noxious stimuli (e.g., pinprick). A small percentage of patients with nerve injury have a nearly pure hypersensitive syndrome in which no sensory deficit is demonstrable. ${ }^{10}$ The quality of the reported sensation may also be a clue: neuropathic pain commonly has a burning or shooting quality with unusual tingling, crawling, or electrical sensations (dysesthesias).

Although none of these characteristics are universally present in neuropathic pain, nor are they absolutely diagnostic of it, their presence does make the diagnosis of neuropathic pain likely.

\section{BEDSIDE ASSESSMENT OF SENSORY SIGNS}

For a precise clinical evaluation, it is important to assess the patients in a standardized way. A sensory bedside examination should include the following qualities: touch, pinprick, pressure, cold, heat, vibration, temporal summation, and after-sensations ${ }^{11,12}$ (Table 2). To assess either a loss (negative sensory signs) or a gain of somatosensory function (positive sensory signs), the responses can be graded as normal, decreased, or increased. The stimulus-evoked (positive) pain types are classified as hyperalgesic or allodynic and according to the dynamic or static character of the stimulus. ${ }^{13}$

Touch can be assessed by gently applying cotton wool to the skin, pinprick sensation by the response to the stimulus of a sharp pinprick, deep pain by gentle pressure on muscle and joints, cold and heat sensation by measuring the response to a thermal stimulus (e.g., metal objects or a glass of water kept at $20^{\circ} \mathrm{C}$ or at $45^{\circ} \mathrm{C}$ ). Cold sensation can also be assessed by the response to acetone spray. Vibration can be assessed by a tuning fork placed at strategic points (e.g., interphalangeal joints). Abnormal temporal summation is the clinical equivalent of increasing neuronal activity after repetitive noxious Cfiber stimulation of $>0.3 \mathrm{~Hz}$. This wind-up-like pain can be produced by mechanical and thermal stimuli. When present, allodynia or hyperalgesia can be quantified by measuring intensity and area. The current general agree- 
Table 2. Definition and Assessment of Negative and Positive Sensory Symptoms and Signs in Neuropathic Pain

\begin{tabular}{|c|c|c|c|}
\hline Symptom or Sign & Definition & Assessment, Bedside Examination & Expected Pathologic Response \\
\hline \multicolumn{4}{|c|}{ Negative signs and symptoms } \\
\hline Hypoesthesia & Reduced sensation to nonpainful stimuli & $\begin{array}{l}\text { Touch skin with paintbrush, cotton swab, or } \\
\text { gauze. }\end{array}$ & Reduced perception, numbness. \\
\hline Pall-hypoesthesia & Reduced sensation to vibration & Apply tuning fork on bone or joint. & Reduced perception. \\
\hline Hypoalgesia & Reduced sensation to painful stimuli & Prick skin with single pin stimulus. & Reduced perception, numbness. \\
\hline Therm-hypoesthesia & Reduced sensation to cold/warm stimuli & $\begin{array}{l}\text { Contact skin with objects at } 10^{\circ} \mathrm{C} \text { (metal } \\
\text { roller, glass with water, coolant such as } \\
\text { acetone). Contact skin with objects at } \\
45^{\circ} \mathrm{C} \text { (metal roller, glass with water). }\end{array}$ & Reduced perception. \\
\hline \multicolumn{4}{|c|}{ Spontaneous sensations or pain } \\
\hline Paresthesia & Nonpainful ongoing sensation (ant crawling). & Grade intensity (0-10). Area in $\mathrm{cm}^{2}$. & - \\
\hline Paroxysmal pain & Shooting electrical attacks for seconds. & $\begin{array}{l}\text { Number per time. Grade intensity }(0-10) \text {. } \\
\text { Threshold for evocation. }\end{array}$ & - \\
\hline Superficial pain & $\begin{array}{l}\text { Painful ongoing sensation often of burning } \\
\text { quality. }\end{array}$ & Grade intensity (0-10). Area in $\mathrm{cm}^{2}$. & - \\
\hline \multicolumn{4}{|l|}{ Evoked pain } \\
\hline $\begin{array}{l}\text { Dynamic mechanical } \\
\text { allodynia }\end{array}$ & $\begin{array}{l}\text { Normally nonpainful light, moving stimuli on skin } \\
\text { evoke pain. }\end{array}$ & $\begin{array}{l}\text { Stroking skin with paintbrush, cotton swab, } \\
\text { or gauze. }\end{array}$ & $\begin{array}{l}\text { Sharp burning superficial pain. Present in } \\
\text { the primary affected zone but spread } \\
\text { beyond into unaffected skin areas } \\
\text { (secondary zone). }\end{array}$ \\
\hline $\begin{array}{l}\text { Static mechanical } \\
\text { allodynia }\end{array}$ & $\begin{array}{l}\text { Normally nonpainful gentle static pressure stimuli } \\
\text { on skin evoke pain. }\end{array}$ & $\begin{array}{l}\text { Manual gentle mechanical pressure at the } \\
\text { skin. }\end{array}$ & $\begin{array}{l}\text { Dull pain. Present in the area of affected } \\
\text { (damaged or sensitized) primary } \\
\text { afferent nerve endings (primary zone). }\end{array}$ \\
\hline $\begin{array}{l}\text { Mechanical punctuate, } \\
\text { pinprick } \\
\text { hyperalgesia }\end{array}$ & $\begin{array}{l}\text { Normally stinging but not painful stimuli evoke } \\
\text { pain. }\end{array}$ & $\begin{array}{l}\text { Manual pricking the skin with a safety pin, } \\
\text { sharp stick, or stiff von Frey filament. }\end{array}$ & $\begin{array}{l}\text { Sharp superficial pain. Present in the } \\
\text { primary affected zone but spread } \\
\text { beyond into unaffected skin areas } \\
\text { (secondary zone). }\end{array}$ \\
\hline Temporal summation & $\begin{array}{l}\text { Repetitive application of identical single noxious } \\
\text { stimuli is perceived as increasing pain sensation } \\
\text { (wind-up-like pain). }\end{array}$ & $\begin{array}{l}\text { Pricking skin with safety pin at interval } \\
<3 \mathrm{~s} \text { for } 30 \mathrm{~s} \text {. }\end{array}$ & $\begin{array}{l}\text { Sharp superficial pain of increasing } \\
\text { intensity. }\end{array}$ \\
\hline Cold allodynia & Normally nonpainful cold stimuli evoke pain. & $\begin{array}{l}\text { Contact skin with objects at } 20^{\circ} \mathrm{C} \text { (metal } \\
\text { roller, glass with water, coolants like } \\
\text { acetone). Control: contact skin with } \\
\text { objects at skin temperature. }\end{array}$ & $\begin{array}{l}\text { Painful often burning temperature } \\
\text { sensation. Present in the area of } \\
\text { affected (damaged or sensitized) } \\
\text { primary afferent nerve endings } \\
\text { (primary zone). }\end{array}$ \\
\hline Heat allodynia & Normally nonpainful heat stimuli evoke pain. & $\begin{array}{l}\text { Contact skin with objects at } 40^{\circ} \mathrm{C} \text { (metal } \\
\text { roller, glass with water). Control: contact } \\
\text { skin with objects at skin temperature. }\end{array}$ & $\begin{array}{l}\text { Painful burning temperature sensation. } \\
\text { Present in the area of affected } \\
\text { (damaged or sensitized) primary } \\
\text { afferent nerve endings (primary zone). }\end{array}$ \\
\hline $\begin{array}{l}\text { Mechanical deep } \\
\text { somatic allodynia }\end{array}$ & $\begin{array}{l}\text { Normally nonpainful pressure on deep somatic } \\
\text { tissues evokes pain. }\end{array}$ & Manual light pressure at joints or muscles. & Deep pain at joints or muscles. \\
\hline
\end{tabular}


ment is that assessment should be conducted in the area of maximal pain, using the contralateral area as control if possible. Nonetheless, contralateral segmental changes after a unilateral nerve or root lesion cannot be excluded, so an examination at mirror sites does not necessarily represent a true control site.

It is important to recognize the spatial distribution of abnormal sensations. In neuropathic conditions, the distinction between primary and secondary area corresponds to the tissue supplied by damaged nerves and the area outside this innervation territory. Mechanical hypersensitivity often expands into the secondary area.

Several sensory signs that can be found in neuropathic pain are defined and the appropriate tests to clinically assess these signs are summarized in Table 2.

\section{ASSESSMENT TOOLS TO DEFINE SUBGROUPS OF PATIENTS WITH SPECIFIC SENSORY PROFILES}

\section{Quantitative sensory testing}

A sophisticated neurophysiologic technique for testing both the nociceptive and non-nociceptive afferent systems in the peripheral and central nervous system is quantitative sensory testing (QST), which uses standardized mechanical and thermal stimuli (e.g., graded von Frey filaments, several pinprick stimuli, pressure algometers, or quantitative thermotesting). Another advantage of QST is that it assesses a loss of function (negative signs) as well as a gain of function (positive signs). Allodynia or hyperalgesia, for example, can be quantified by mea-

A
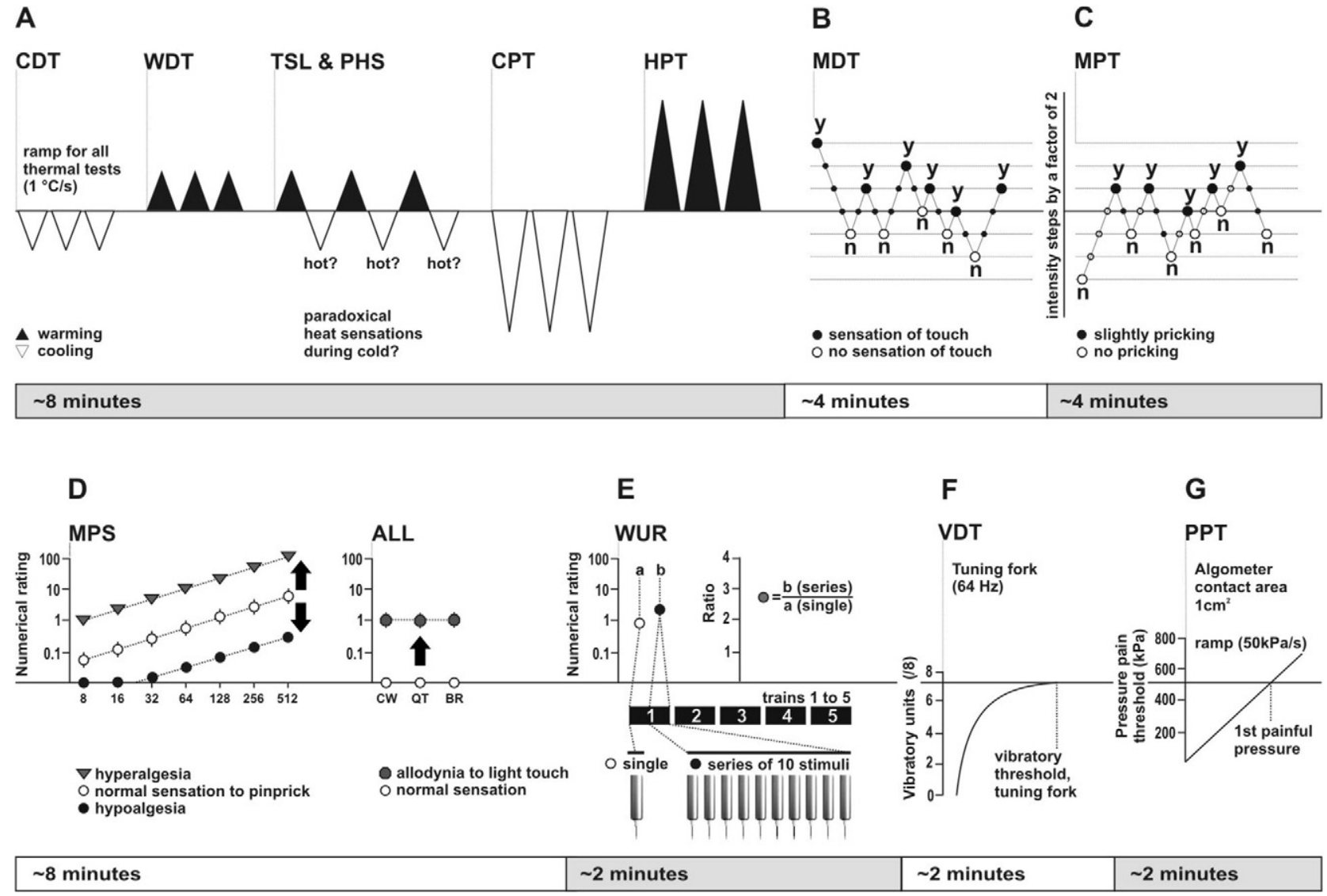

FIG. 1. Methods in the quantitative sensory testing (QST) battery of sensory tests. The standardized QST protocol assesses 13 variables in seven test procedures. All procedures presented include a time frame for testing over one area. (A) Thermal testing comprises detection and pain thresholds for cold, warm, or hot stimuli, mediated by C- and A-delta fibers. (B) Tests for A-beta fiber function using von Frey filaments. (C) Tests for A-delta fiber mediated hyper- or hypoalgesia to pinprick stimuli. (D) (stimulus/responsefunctions: MPS for pinprick stimuli and ALL for dynamic mechanical allodynia); assesses again A-delta mediated sensitivity to sharp stimuli (pinprick) and also A-beta fiber mediated sensitivity to stroking light touch. (E) compares the verbal ratings within 5 trains of a single pinprick stimulus $a$ with a series $b$ of 10 repetitive pinprick stimuli to calculate WUR as the ratio b/a. (F) Tests again for A-beta fiber function using a Rydel-Seiffer $64 \mathrm{~Hz}$ tuning fork. (G) The only test for deep pain sensitivity, most probably mediated by muscle Cand A-delta fibers. ALL = dynamic mechanical allodynia; BR = brush; CDT = cold detection threshold; CPT = cold pain threshold; $\mathrm{CW}=$ cotton wisp (cotton ball); QT = cotton wool tip (cotton-tipped swab); HPT = heat pain threshold; MDT = mechanical detection threshold; MPS = mechanical pain sensitivity; MPT = mechanical pain threshold; $n=n o ; ~ P H S=$ number of paradoxical heat sensations during the TSL procedure; PPT = pressure pain threshold; TSL = thermal sensory limen for alternating warm and cold stimuli; VDT = vibration detection threshold); WDT = warm detection threshold; WUR = wind-up ratio; y = yes. (Figure after Rolke et al., ${ }^{14}{ }^{2006 .)}$ 
suring intensity, threshold for elicitation, duration, and area.

A standardized protocol for QST was recently proposed by the nationwide German Network on Neuropathic Pain (DFNS $)^{14}$; it includes 13 parameters of sensory testing procedures for the analysis of the exact somatosensory phenotype of neuropathic pain patients (FIG. 1). To evaluate pathologic ranges of positive or negative signs in patients, an age- and sex-matched database for absolute and relative QST reference data was established for healthy human subjects. To date, this nationwide multicenter trial has complete sensory profiles of 180 healthy human subjects and more than 1000 neuropathic pain patients of a variety of entities. Thermal detection and pain thresholds are determined, including a test for the presence of paradoxical heat sensations, mechanical detection thresholds to von Frey filaments and a $64 \mathrm{~Hz}$ tuning fork, mechanical pain thresholds to pinprick stimuli and blunt pressure, stimulus-response functions for pinprick and dynamic mechanical allodynia (pain to light touch), and pain summation (wind-up ratio) using repetitive pinprick stimulation. Data for healthy human subjects were analyzed for the influence of body side and region, age, and sex. Using the DFNS protocol for most variables, pathologic values of positive and negative signs can be detected on the basis of reference data. An analysis of 1236 patients with neuropathic pain of various etiologies revealed the exact frequency of

Table 3. Frequency of Pathologic Values in Percent (Gain and Loss) for Complex Regional Pain Syndromes (CRPS, $n=403)$ and Polyneuropathies (PNP, $n=343)$

\begin{tabular}{lrrrrr}
\hline & \multicolumn{2}{c}{ Gain } & & \multicolumn{2}{c}{ Loss } \\
\cline { 2 - 3 } \cline { 5 - 6 } & CRPS & PNP & & CRPS & PNP \\
\hline CDT & 2.7 & 0.3 & & 32.5 & 40.2 \\
WDT & 2.5 & 0.9 & & 26.6 & 18.4 \\
TSL & 2.7 & 0.6 & & 26.9 & 36.7 \\
CPT & 30.5 & 1.5 & & 5.2 & - \\
HPT & 40.1 & 7.0 & & 7.7 & 5.0 \\
PPT & 66.3 & 5.0 & & 3.3 & 13.2 \\
MPT & 28.7 & 11.1 & 10.0 & 21.9 \\
MPS & 46.6 & 8.5 & 6.2 & 5.0 \\
WUR & 13.1 & 6.9 & & 2.7 & 0.4 \\
MDT & 9.5 & 0.3 & 35.2 & 39.8 \\
VDT & 1.5 & - & 35.4 & 45.9 \\
PHS & 9.4 & 37.3 & - & - \\
DMA & 24.1 & 12.0 & - & - \\
\hline
\end{tabular}

$\mathrm{CDT}=$ cold detection threshold; $\mathrm{CPT}=$ cold pain threshold; DMA = dynamic mechanical allodynia; HPT = heat pain threshold; MDT = mechanical detection threshold; MPS $=$ mechanical pain sensitivity; MPT = mechanical pain threshold; PHS = number of paradoxical heat sensations during the TSL procedure; $\mathrm{PPT}=$ pressure pain threshold; TSL $=$ thermal sensory limen for alternating warm and cold stimuli; VDT $=$ vibration detection threshold; WDT $=$ warm detection threshold; WUR = wind-up ratio.

Source: Maier et al., ${ }^{15} 2008$. somatosensory abnormalities in the affected skin area. ${ }^{15}$ Table 3 shows an example of these data for two neuropathic entities. These data are of utmost importance for the design of drug trials in which compounds are tested that are known to target specific sensory signs.

There is nonetheless one caveat with an approach that addresses only single sensory signs. Animal research has shown that it is probably not appropriate to link one single sign to exactly one underlying pathophysiological mechanism. Rather, one specific sign may be generated by several entirely different underlying pathophysiological mechanisms. Thus, it became clear that a specific constellation of sensory signs (a profile of signs; i.e., a combination of negative and positive sensory phenomena) might be able to predict the mechanisms more precisely than just one single parameter.

To translate these ideas into a clinical framework, it is important to characterize the heterogeneity of the individual somatosensory phenotype as precisely as possible. For example, the standardized QST method described can nicely distinguish between phenotypic subtypes of postherpetic neuralgia patients with distinct sensory symptom constellations ${ }^{14}$ (FIG. 2). The profile of patient 1 shows a predominant gain of sensory function in terms of heat pain hyperalgesia (HPT), pinprick mechanical hyperalgesia (MPS), dynamic mechanical allodynia (ALL), and static hyperalgesia to blunt pressure (PPT). In terms of mechanisms, this profile is consistent with a combination of peripheral and central sensitization. The QST profile of patient 2 shows a predominant loss of sensory function. The cold (CDT) and warm (WDT) detection thresholds, thermal sensory limen (TSL), heat pain thresholds (HPT), tactile detection thresholds (MDT), and mechanical pain thresholds to pinprick stimuli (MPT) are outside the normal range in the sense of a loss of function. This profile is consistent with a combined small fiber and large fiber sensory deafferentation. (Notably, both patients experienced spontaneous burning pain of similar intensity.)

\section{Patient reported outcomes}

The exact QST profiling using 13 somatosensory tests takes more than 1 hour of the investigator's and the patient's time. Thus, for practicability this approach can be used only in a research setting and in relatively small proof-of-concept trials. In large clinical phase III trials, alternative easy-to-use assessment strategies are essential. Furthermore, pain is a subjective phenomenon, and it is still not quite clear whether QST parameters (e.g., a pain perception threshold to a small thermal stimulus) can give a real picture of a patient's subjective experience and whether these parameters are therefore clinically relevant as perceived by the patients themselves. ${ }^{16,17}$ Alternatively, the question arises whether patient-reported outcomes, which collect health-related data directly from the patient, ${ }^{18} \mathrm{can}$ 


\section{O PHN I: Peripheral and central sensitization - PHN II: Deafferentation of small and large fibers}

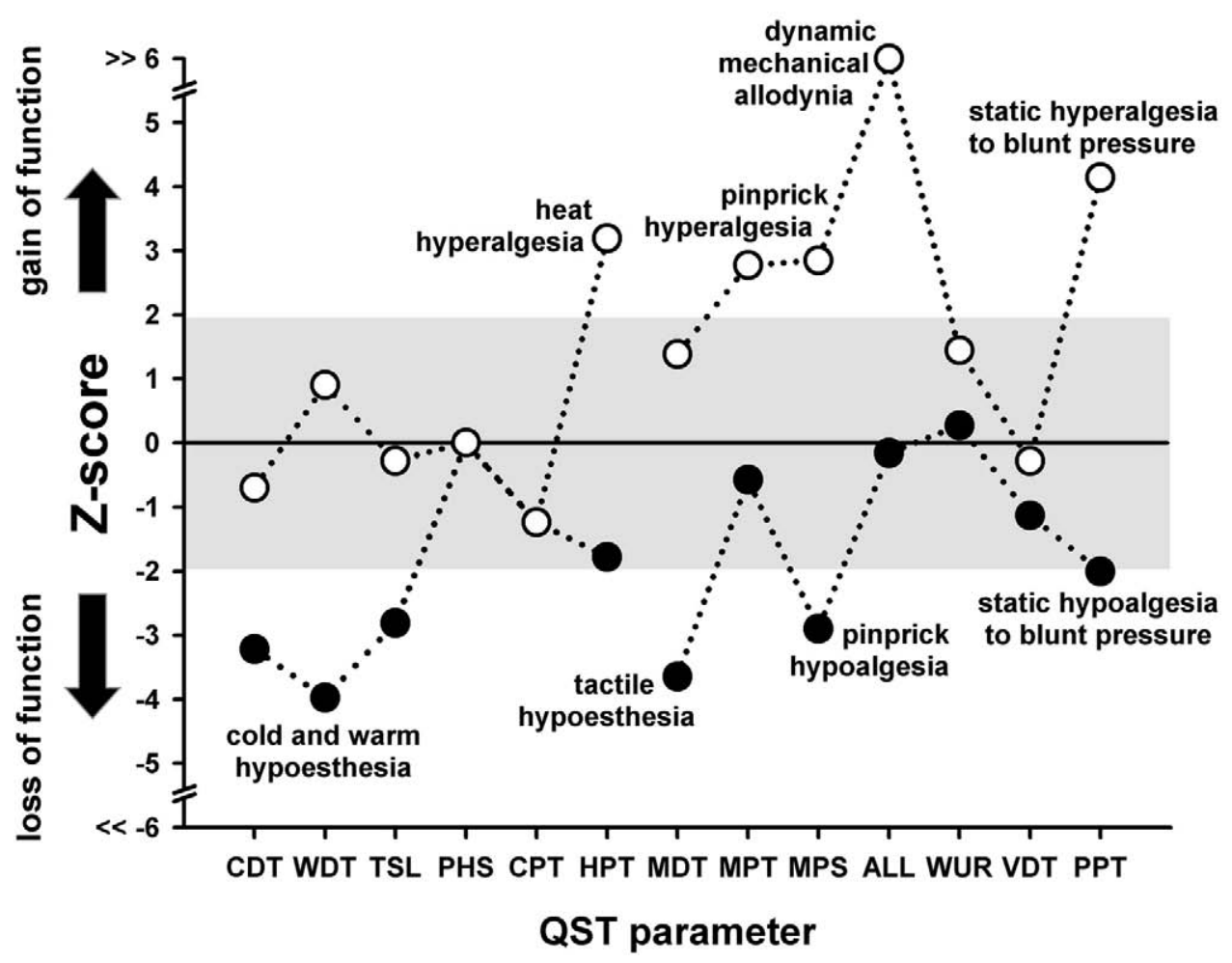

FIG. 2. Z-score sensory profiles of two patients with postherpetic neuralgia (PHN). Patient PHN I presents the QST profile of a 70 -year-old woman who has had PHN for 8 years. Ongoing pain was 80 on a $0-100$ numeric rating scale. The profile shows a predominant gain of sensory function in terms of heat pain hyperalgesia (HPT), pinprick mechanical hyperalgesia (MPS), dynamic mechanical allodynia (ALL), and static hyperalgesia to blunt pressure (PPT) outside the 95\% confidence interval of the distribution of healthy subjects (gray zone). This profile is consistent with a combination of peripheral and central sensitization. Patient PHN II shows the QST profile of a 71-year-old woman with pain for 8 months. Ongoing pain was 70 on a 0-100 numeric rating scale. The QST profile shows predominant loss of sensory function. Note the cold (CDT) and warm (WDT) detection thresholds, thermal sensory limen (TSL), heat pain thresholds (HPT), tactile detection thresholds (MDT), and mechanical pain thresholds to pinprick stimuli (MPT) outside the normal range (gray zone). This profile is consistent with a combined small and large fiber sensory deafferentation. Z-score: Numbers of standard deviations between patient data and group-specific mean value. (Figure after Rolke et al., ${ }^{14} 2006$.)

capture subtle differences in individual somatosensory characteristics.

It was recently confirmed that patient-reported outcomes can be used to classify neuropathic pain on the basis of the patients' perceived sensory neuropathic symptoms. The painDETECT questionnaire comprises questions regarding the severity, course, quality, and nature of the patient's pain and the specific neuropathic pain symptoms ${ }^{19}$ (Table 2). In particular, the nine questions address the following sensory symptoms: 1) spontaneous burning pain; 2) spontaneous paresthesias; 3) dynamic mechanical allodynia; 4) spontaneous pain attacks; 5) thermal hyperalgesia; 6) numbness; 7) deep somatic allodynia; 8) pain course pattern; 9) pain radiating.

Using this questionnaire, 498 patients with postherpetic neuralgia and 1623 patients with painful diabetic polyneuropathy were analyzed. ${ }^{20}$ To identify relevant subgroups of patients who are characterized by a char- acteristic symptom profile, a hierarchical cluster analysis was performed for this cohort. The clusters are represented by the patterns of questionnaire scores, which thus shows the typical pathologic structure of the respective group. With this approach, five distinct clusters or subgroups of patients could be detected that show a characteristic sensory profile (i.e., a typical constellation and combination of neuropathic symptoms) (FIG. 3). The sensory profiles show remarkable differences in the expression of the symptoms. All subgroups occur in relevant numbers in both disease entities, but the frequencies differ between postherpetic neuralgia and painful diabetic polyneuropathy. From such patterns, it is possible to speculate about the underlying pathophysiological mechanisms that potentially operate in these phenotypic subgroups.

Subgroup 1 occurs nearly three times more frequently in postherpetic neuralgia than in painful diabetic neuropathy. The prominent features in this subgroup are mod- 


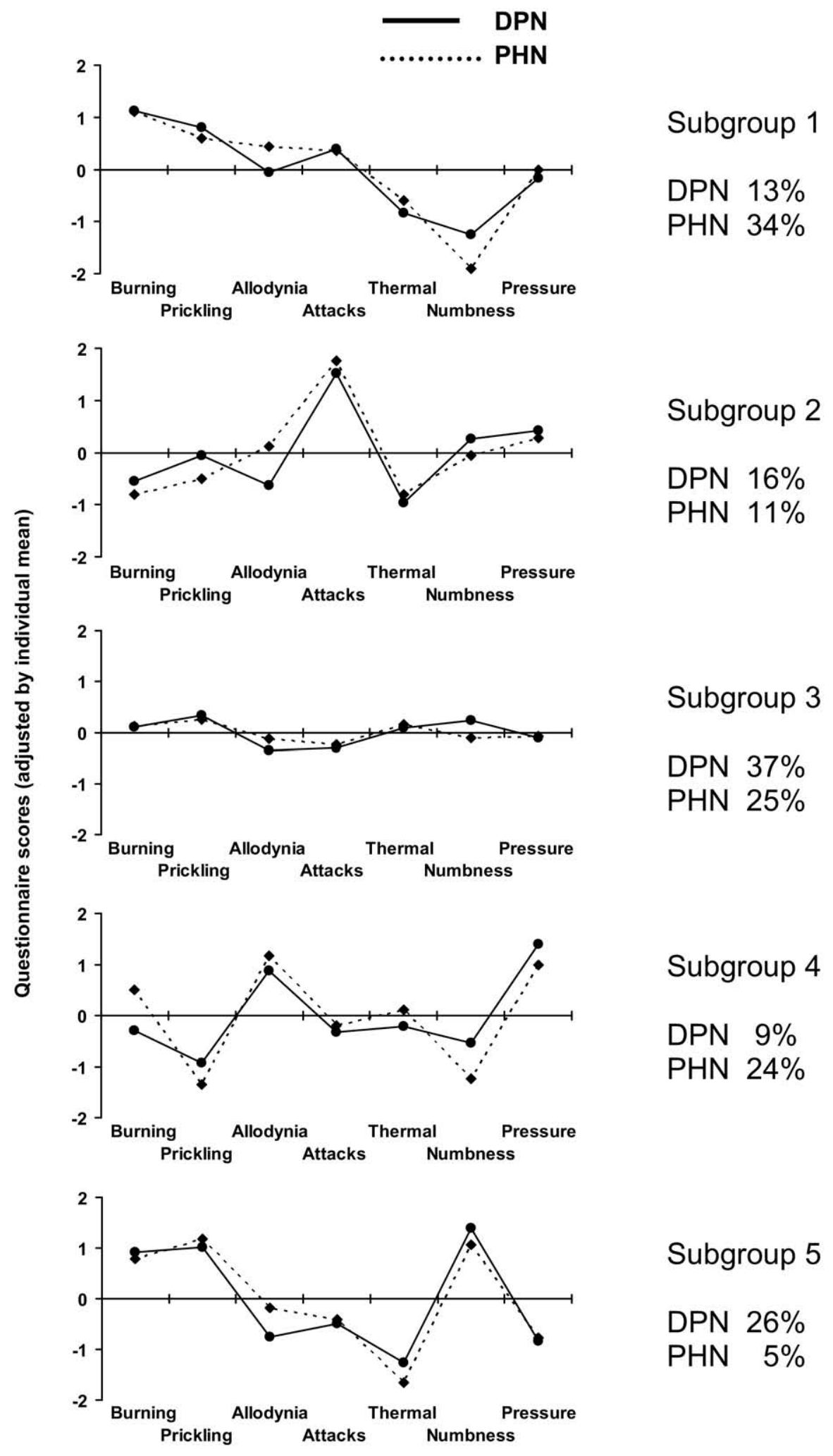

erate to strong spontaneous burning pain, in combination with slight to moderate dynamic mechanical allodynia, the latter being more intense in postherpetic neuralgia than in diabetic neuropathy. Numbness was almost never noticed, which indicates a preserved cutaneous innervation without any signs of degeneration. This profile might reflect preserved and irritable nociceptors with secondary sensitization of central nociceptive neurons. 
Pharmacologic compounds that modulate neuronal sensitization could be used favorably in this group. Interestingly, the sensory perceptions of this group of patients closely represent the constellation of sensory signs that have been described with QST in a subset of patients (FIG. 2).

Subgroup 2 is characterized by clinically relevant pain attacks. These patients could be preferably included in trials with drugs that are known to be effective in reducing ectopic neuronal firing in nociceptors, such as sodium channel blockers.

Patients in subgroup 4 (subgroup 3 is considered separately, below) demonstrate a combination of considerable dynamic mechanical allodynia and deep somatic hyperalgesia. This profile could be explained by a predominant involvement of afferents innervating deep somatic structures. Cutaneous allodynia might be due to a convergent afferent input of deep somatic and skin nerves on central nociceptive neurons. In this subgroup 4 , compounds that interfere with central sensitization could be more successful.

Patients in subgroup 5 experience considerable burning pain and paresthesias, but do not have relevant dynamic mechanical allodynia, thermal hyperalgesia, or pain attacks. Numbness, however, is very prominent. This profile indicates a severe deafferentation of the affected skin. A length-dependent denervation of afferent neurons (which occurs more often in diabetic neuropathy) nicely explains these findings. This cohort might be most appropriate for trials with drugs that are predicted to be effective on spontaneous afferent sensations rather than on evoked pain types. In this case, the perception profile reflects the QST findings of patient 2 (FIG. 2).

A somewhat unexpected finding was that in subgroup 3, a large subgroup (one third of the entire cohort), the values of the sensory profile are mainly concentrated around the zero-line (moderate perception) for all parameters. This finding could be interpreted in several ways. First, a large group of patients might perceive all neuropathic symptoms in a similar frequency and intensity. From clinical experience and quantitative sensory testing studies, it is unlikely that $30 \%$ of the patients belong to this group. Second, a psychological phenomenon (i.e., the tendency to score all questions similarly or identically) could be responsible for this type of profile. These patients might be, for whatever reason, unable to discriminate among the different sensory abnormalities and would thus answer all questions equally. This population should probably be excluded from clinical trials in which the effect of a compound on specific symptoms is evaluated.

\section{Interview and bedside examination}

In a recent study, a combination of neuropathic symptoms as well as signs was assessed using a structured interview and a standardized bedside examination in 130 patients with painful diabetic polyneuropathy, postherpetic neuralgia, or radicular back pain and in 57 patients with non-neuropathic low back pain. ${ }^{21}$ The interview consisted of 16 questions exploring 46 items covering pain localization, evoked pains, and pain quality, along with further sensory qualities such as dysesthesia and numbness. The physical examination included 23 bedside tests that provided information about 39 items covering trophic and autonomic cutaneous signs, evoked pains, and sensory deficits using von Frey filaments, blunt pressure, brush, vibration, pinprick, temperature, passive movement, and the straight-leg-raising test. With this approach, six subgroups of patients with neuropathic pain and two subgroups of patients with non-neuropathic pain could be distinguished. The physical examination was shown to be more important for the distinction of pain subtypes than symptoms assessed by the interview.

\section{CAVEATS}

As already noted, the modern theoretical concept of a mechanism-based therapy relies on the assumption that specific sensory profiles predict specific underlying mechanisms. It should be possible for a clinician to identify a particular mechanism by assessing a specific sensory symptom profile. However attractive a subtype classification based on the afferent profiles might be, it should be emphasized that not all patients fit exactly into one subgroup or another. It is rather to be seen as a statistical approximation of a best-fit to a particular subgroup.

Furthermore, in some individual patients many heterogeneous patterns of sensory dysfunction can be detected in adjacent cutaneous spots. ${ }^{22}$ Accordingly, it could be shown that detailed testing of sensory function, chemical

\footnotetext{
FIG. 3. Subgrouping of painful diabetic polyneuropathy (DPN) and postherpetic neuralgia (PHN) patients according to sensory profiles using patient-reported outcomes. To identify relevant subgroups of patients who are characterized by a characteristic symptom constellation a hierarchic cluster analysis was performed. The clusters are represented by the patterns of questionnaire scores (adjusted individual mean), thus showing the typical pathologic structure of the respecting group. With this approach, five clusters (subgroups) with distinct symptom profiles could be detected. Sensory profiles show remarkable differences in the expression of symptoms. ${ }^{20}$ Adjusted individual mean: To eliminate interindividual differences in general perception of sensory stimuli (differences in individual pain perception thresholds), an adjusted score was calculated in which the mean of the 0-5 values marked for all seven questions was subtracted from the given 0-5 score for each question. In this individual score, values greater than 0 indicate a sensation that is more intense than the individual mean pain perception, and values less than 0 indicate a sensation that is less intense than the individual mean pain perception. DPN $=$ diabetic painful neuropathy; PHN = postherpetic neuralgia; \% = frequency of occurrence. (Figure after Baron et al., ${ }^{20} 2009$. )
} 
stimulation with capsaicin, and assessment of epidermal innervation density in patients with postherpetic neuralgia clearly showed areas of relative preservation of sensory functions in close vicinity to impaired thermal sensation, both within the affected dermatome. ${ }^{23}$ Moreover, sensory profiles may vary considerably over the time course of the disease; most frequently, a switch from hypersensitivity to deafferentation is noted. The latter might be in line with the observation that some patients show a favorable treatment response early in the disease course but become more and more refractory along with the development of chronicity.

Nonetheless, with classification of patients based on the sensory perception thresholds within the most painful skin area it is possible to detect the predominant individual sensory profile and the most likely underlying pain-generating mechanism.

Another caveat addressing the predictive value of QST measurements for therapy was recently revealed by two independent studies. It was hypothesized that topically applied lidocaine, which is believed to act on ectopic discharges in hyperactive nociceptive fibers, would be beneficial in particular for patients with sensitized peripheral nociceptors, compared with patients with a loss of dermal nociceptors. In negation of the hypothesis, however, skin biopsies and QST could not identify lidocaine responders in painful neuropathies ${ }^{24}$ and postherpetic neuralgia. ${ }^{25}$ This disappointing finding yet once more underscores the notion that a precise analysis of the entire scenario of the individual somatosensory perception - the whole variety of sensory abnormalities-might be more appropriate for mapping the underlying mechanisms and to be used as a predictor of response.

\section{CONCLUSION}

The approach of classifying and subgrouping patients with neuropathic pain on the basis of symptoms or signs opens up new possibilities for stratifying patients in clinical trials on the basis of sensory profiles and, thus, in terms of the potential underlying mechanisms. QST analyses or results of questionnaires can be used to calculate a priori to which subgroup a particular patient belongs with the highest probability. The expected benefits of such a selection are twofold. First, in clinical proof-ofconcept trials the study population could be enriched prospectively on the basis of a priori defined entry criteria. This enrichment with patients who potentially require a specific treatment should increase the likelihood for statistically valid and positive trial outcomes. Second, in clinical practice, it would be possible to establish an individualized therapy: that is, to identify the particular patients who require a specific treatment option.
Acknowledgments: This work was supported by the German Ministry of Research and Education, German Research Network on Neuropathic Pain (BMBF, 01EM01/04), the German Ministry of Research and Education, Modelling Pain Switches (BMBF, 03154493), and an unrestricted educational grant from Pfizer and Grünenthal, Germany.

\section{REFERENCES}

1. Treede RD, Jensen TS, Campbell JN, et al. Neuropathic pain: redefinition and a grading system for clinical and research purposes. Neurology 2007;70:1630-1635.

2. Jensen TS, Baron R. Translation of symptoms and signs into mechanisms in neuropathic pain. Pain 2003;102:1-8.

3. Baron R. Mechanisms of disease: neuropathic pain-a clinical perspective. Nat Clin Pract Neurol 2006;2:95-106.

4. Baron R, Binder A. How neuropathic is sciatica? The mixed pain concept [In German]. Orthopade 2004;33:568-575.

5. Freynhagen R, Rolke R, Baron R, et al. Pseudoradicular and radicular low-back pain: a disease continuum rather than different entities? Answers from quantitative sensory testing. Pain 2007;135:65-74.

6. Dworkin RH, O'Connor AB, Backonja M, et al. Pharmacologic management of neuropathic pain: evidence-based recommendations. Pain 2007;132:237-251.

7. Vinik AI, Tuchman M, Safirstein B, et al. Lamotrigine for treatment of pain associated with diabetic neuropathy: results of two randomized, double-blind, placebo-controlled studies. Pain 2007;128:169179.

8. Katz J, Finnerup NB, Dworkin RH. Clinical trial outcome in neuropathic pain: relationship to study characteristics. Neurology 2008;70:263-272.

9. Woolf CJ, Bennett GJ, Doherty M, et al. Towards a mechanismbased classification of pain? Pain 1998;77:227-229.

10. Fields HL, Rowbotham M, Baron R. Postherpetic neuralgia: irritable nociceptors and deafferentation. Neurobiol Dis 1998;5:209-227.

11. Bouhassira D, Attal N, Fermanian J, et al. Development and validation of the Neuropathic Pain Symptom Inventory. Pain 2004; 108:248-257.

12. Cruccu G, Anand P, Attal N, et al. EFNS guidelines on neuropathic pain assessment. Eur J Neurol 2004;11:153-162.

13. Rasmussen PV, Sindrup SH, Jensen TS, Bach FW. Symptoms and signs in patients with suspected neuropathic pain. Pain 2004;110: 461-469.

14. Rolke R, Baron R, Maier C, et al. Quantitative sensory testing in the German Research Network on Neuropathic Pain (DFNS): standardized protocol and reference values [Erratum in: Pain 2006; 125:197]. Pain 2006;123:231-243.

15. Maier $\mathrm{C}$, Richter $\mathrm{H}$, Baron R. A new classification of neuropathic pain (LoGa) [In German]. Schmerz 2008;22 Suppl 2:74 (abstract).

16. McDermott AM, Toelle TR, Rowbotham DJ, Schaefer CP, Dukes EM. The burden of neuropathic pain: results from a cross-sectional survey. Eur J Pain 2006;10:127-135.

17. Davies M, Brophy S, Williams R, Taylor A. The prevalence, severity, and impact of painful diabetic peripheral neuropathy in type 2 diabetes. Diabetes Care 2006;29:1518-1522.

18. Acquadro C, Berzon R, Dubois D, et al.; PRO Harmonization Group. Incorporating the patient's perspective into drug development and communication: an ad hoc task force report of the Patient-Reported Outcomes (PRO) Harmonization Group meeting at the Food and Drug Administration, February 16, 2001. Value Health 2003;6:522-531.

19. Freynhagen R, Baron R, Gockel U, Tölle TR. painDETECT: a new screening questionnaire to identify neuropathic components in patients with back pain. Curr Med Res Opin 2006;22:1911-1920.

20. Baron R, Tölle TR, Gockel U, Brosz M, Freynhagen R. A cross-sectional cohort survey in 2100 patients with painful diabetic neuropathy and postherpetic neuralgia: differences in demographic data and sensory symptoms. Pain 2009 [Epub ahead of print, July 8].

21. Scholz J, Mannion RJ, Hord DE, et al. A novel tool for the assessment of pain: validation in low back pain. PLoS Med 2009; 6:e1000047. 
22. Pappagallo M, Oaklander AL, Quatrano-Piacentini AL, Clark MR, Raja SN. Heterogenous patterns of sensory dysfunction in postherpetic neuralgia suggest multiple pathophysiologic mechanisms. Anesthesiology 2000;92:691-698.

23. Petersen KL, Fields HL, Brennum J, Sandroni P, Rowbotham MC. Capsaicin evoked pain and allodynia in post-herpetic neuralgia. Pain 2000;88:125-133.
24. Herrmann DN, Pannoni V, Barbano RL, Pennella-Vaughan J, Dworkin RH. Skin biopsy and quantitative sensory testing do not predict response to lidocaine patch in painful neuropathies. Muscle Nerve 2006;33:42-48.

25. Wasner G, Kleinert A, Binder A, Schattschneider J, Baron R. Postherpetic neuralgia: topical lidocaine is effective in nociceptordeprived skin. J Neurol 2005;252:677-686. 